\title{
Feeding Difficulties in Children: A Guide for Allied Health Professionals An Evaluation Report
}

\author{
Carmel M. Blayden \\ NSW Children's Healthcare Network, Carmel.Blayden@health.nsw.gov.au \\ Kate Holland \\ Sonia Hughes \\ NSW Children's Healthcare Network, Sonia.Hughes@health.nsw.gov.au \\ Jennifer Nicol \\ NSW Children's Healthcare Network, jennifer.nicol@health.nsw.gov.au
}

Child \& Family Health Services, Community Health, Sydney Local Health, Kate.Holland@health.nsw.gov.au

Follow this and additional works at: https://nsuworks.nova.edu/ijahsp

Part of the Medicine and Health Sciences Commons

\section{Recommended Citation}

Blayden CM, Holland K, Hughes S, Nicol J. Feeding Difficulties in Children: A Guide for Allied Health Professionals An Evaluation Report. The Internet Journal of Allied Health Sciences and Practice. 2021 Jan 01;19(3), Article 16.

This Manuscript is brought to you for free and open access by the College of Health Care Sciences at NSUWorks. It has been accepted for inclusion in Internet Journal of Allied Health Sciences and Practice by an authorized editor of NSUWorks. For more information, please contact nsuworks@nova.edu. 


\title{
Feeding Difficulties in Children: A Guide for Allied Health Professionals An Evaluation Report
}

\begin{abstract}
Purpose: An evaluation of Feeding Difficulties in Children: A Guide for Allied Health Professionals (2016) was undertaken to determine how the publication has been utilised amongst allied health professionals across NSW. This evaluation aimed to identify the usefulness of the Guide in supporting the assessment and management of children with feeding difficulties. Results will inform future education and support strategies. Method: The evaluation targeted allied health professionals employed by NSW Health. An online questionnaire formed the primary method of data collection. Four semi-structured focus groups also occurred to provide complementary qualitative information. Results: Clinicians from a range of professions and healthcare settings responded to the online questionnaire $(\mathrm{N}=100)$ and participated in focus groups $(N=15)$, reflecting the range of allied health professionals who work with children with feeding difficulties. Qualitative and quantitative data obtained identified a number of key themes. The data collected provided a robust overview of how the Guide has been utilised. The main findings relate to five key themes: characteristics of respondents and their teams; use of the Guide; knowledge of content; satisfaction with the Guide; and future directions. Conclusions: The evaluation indicated that the Guide is viewed as a useful tool by allied health professionals working with children with feeding difficulties. Experienced respondents reported that the Guide provided validation for current practice whereas those with less experience indicated it supported their work within this clinically complex realm. Further promotion of the Guide and multi-modal education regarding its use is recommended.
\end{abstract}

\section{Author Bio(s)}

Carmel Blayden, MHPE, MHIthSc, BAppSc(OT), is an Allied Health Educator with the NSW Children's Healthcare Network.

Kate Holland, MNutrDiet,BAppSc(SpPath), is a Senior Dietitian, Child \& Family Community Health, Sydney Local Health District.

Sonia Hughes, BAppSc(SpPath), is an Allied Health Educator with the NSW Children's Healthcare Network.

Jennifer Nicol, MEd(HIthProf), MHA, BAppSc(SpPath), is an Allied Health Educator with the NSW Children's Healthcare Network.

\section{Acknowledgements}

We would like to acknowledge the work of the allied health professionals working with children with feeding difficulties. 


\title{
TIJAHSP" \\ The Internet Journal of Allied Health Sciences and Practice
}

Vol. 19 No. 3 ISSN 1540-580X

\section{Feeding Difficulties in Children - A Guide for Allied Health Professionals: An Evaluation Report}

\author{
Carmel M. Blayden ${ }^{1}$ \\ Kate Holland2 \\ Sonia Hughes ${ }^{1}$ \\ Jennifer Nicol ${ }^{1}$
}

1. NSW Children's Healthcare Network

2. Child \& Family Community Health, Sydney Local Health District

Australia

\begin{abstract}
Purpose: An evaluation of Feeding Difficulties in Children - A Guide for Allied Health Professionals (2016) was undertaken to determine how the publication has been utilised amongst allied health professionals across NSW. This evaluation aimed to identify the usefulness of the Guide in supporting the assessment and management of children with feeding difficulties. Results will inform future education and support strategies. Method: The evaluation targeted allied health professionals employed by NSW Health. An online questionnaire formed the primary method of data collection. Four semi-structured focus groups also occurred to provide complementary qualitative information. Results: Clinicians from a range of professions and healthcare settings responded to the online questionnaire $(\mathrm{N}=100)$ and participated in focus groups $(\mathrm{N}=15)$, reflecting the range of allied health professionals who work with children with feeding difficulties. Qualitative and quantitative data obtained identified a number of key themes. The data collected provided a robust overview of how the Guide has been utilised. The main findings relate to five key themes: characteristics of respondents and their teams; use of the Guide; knowledge of content; satisfaction with the Guide; and future directions. Conclusions: The evaluation indicated that the Guide is viewed as a useful tool by allied health professionals working with children with feeding difficulties. Experienced respondents reported that the Guide provided validation for current practice whereas those with less experience indicated it supported their work within this clinically complex realm. Further promotion of the Guide and multi-modal education regarding its use is recommended.
\end{abstract}

Keywords: feeding difficulties, allied health, children, paediatric feeding 


\section{INTRODUCTION}

Feeding is a process dependent on many complex systems. It encompasses various facets of a child's development including oral motor skills, nutritional requirements, environment, interaction with carers, and sensory processing. ${ }^{1-2}$ Feeding difficulties commonly develop as a result of a range of circumstances early in life and may present as a difficulty at any level of the feeding process. Therefore, assessment and intervention for infants and children with feeding difficulties involves significantly more consideration than a clinical observation of a feed. ${ }^{3}$ Health care professionals need a systematic approach to assessing and managing feeding difficulties. ${ }^{4} \mathrm{An}$ interdisciplinary feeding team approach allows for coordinated global assessment and management. ${ }^{4-5}$

Feeding Difficulties in Children - A Guide for Allied Health Professionals (hereafter referred to as "the Guide"), was published in 2016 by the then Office of Kids and Families. ${ }^{1}$ The Guide was developed in response to a need identified by New South Wales (NSW) Health allied health professionals working with children. These clinicians requested further education and guidance with regards to the assessment and management of children with feeding difficulties. ${ }^{1}$

The primary value of guidelines is that they strengthen the quality of care received by patients by improving the clinical decision-making process. ${ }^{6}$ The aim of the Guide, therefore, was to provide recommendations, information, and guidance to support the clinical decision making of allied health professionals in services provided by NSW Health for the assessment and management of children with feeding difficulties.

This study aimed to determine how the Guide has been utilised amongst allied health professionals in different settings across NSW. In addition, the evaluation also aimed to identify the usefulness of the Guide in supporting the assessment and management of children with feeding difficulties. Results of the evaluation will be reported to key stakeholders. The NSW Children's Healthcare Network Allied Health Educators will use the recommendations to inform future education and support related to the assessment and management of paediatric feeding difficulties in children.

\section{METHODOLOGY}

Allied health professionals from a range of backgrounds and experience provide services for children with feeding difficulties. Clinical practice suggests that children experiencing feeding difficulties are an increasing caseload for allied health professionals across NSW. ${ }^{1}$ There is significant variability in type and level of paediatric service provision for this client group. Evaluation of the use of the Guide, therefore, targeted allied health professionals employed by NSW Health as part of a quality improvement project. The quality improvement plan was approved and reviewed throughout the process by the NSW Children's Healthcare Network Allied Health Education Advisory Committee.

Quantitative and qualitative data was collected using an online questionnaire and focus groups. These measures explored the use of the Guide and the perceptions of the allied health professionals. It also investigated the extent to which the Guide was able to provide recommendations, information, and guidance to support clinical decision making and identify future enhancements. Participants in the evaluation were recruited via an email forwarded to allied health professionals working with children across NSW. The email included both the link to the questionnaire and a call for interested clinicians to participate in a focus group. Participation in all aspects of the evaluation was voluntary and information de-identified.

The questionnaire formed the primary method of data collection as it enabled open and closed standardised questions to be asked to a large number of targeted allied health professionals. The questionnaire was constructed to gather information on the attitudes and behaviours of allied health professionals using the Guide. The planning and construction of the survey instrument utilised the major steps outlined by numerous authors. ${ }^{6-8}$ The questionnaire was based on the conceptual framework of the Guide with each question reviewed by the authors for relevance and clarity and to ensure that the correct type of question was posed for each component. The final tool included questions relating to the sub-topics of demographics, feeding team details, general feeding experience, implementation of the Guide, satisfaction, assessment of behaviour change, future directions, and opportunities for training. These questions were piloted with a range of allied health professionals from across NSW who had an interest in the Guide to confirm validity of the tool.

In addition to the questionnaire, four focus groups occurred using a semi-structured interview format. The open-ended interview approach allowed collation of more in-depth information that may not have been included in the questionnaire. ${ }^{9}$ Each focus group included between three and four participants comprising a mix of clinicians from different professional backgrounds, levels of experience, and health facilities. Focus groups occurred via videoconference and/or teleconference with the purpose of providing in-depth, qualitative information to complement the data collected from the questionnaire. Participants in the focus groups were asked semi-structured questions relating to their use of the Guide in their service, how it has changed their practice, areas for improvement, and ways in which allied health professionals working with children with feeding difficulties could be better supported. Group sessions were recorded to enable accurate transcription of responses. Recordings were then deleted. 
Qualitative data for all outcomes and results were compared between sub groups. Summary statistics are reported as frequency and percentages. Thematic analysis was used to identify themes within the open ended questions. This analysis was completed using the sixphase guide described by Braun and Clarke. ${ }^{10}$

\section{RESULTS}

Results of the survey were collated and discussed under the headings a) demographics; b) feeding caseload and service provision; c) implementation of the Guide; $d$ ) content of the Guide; and e) impact of the Guide. Comparative analysis of the individual professional groups was not conducted due to variations in the populations completing the survey. A number of key themes were identified by the focus groups including use of the Guide, areas for improvement, change in practice, support for the use of the Guide, and further education and support. These key themes are discussed under the appropriate survey heading.

\section{Demographics}

Respondents $(\mathrm{N}=100)$ reflect the range of allied health professionals working with children with feeding difficulties. Figure 1 displays the distribution of respondents by health profession. These professionals represent 14 NSW Health Local Health Districts (LHD), Sydney Children's Hospitals Network, and ACT Health.

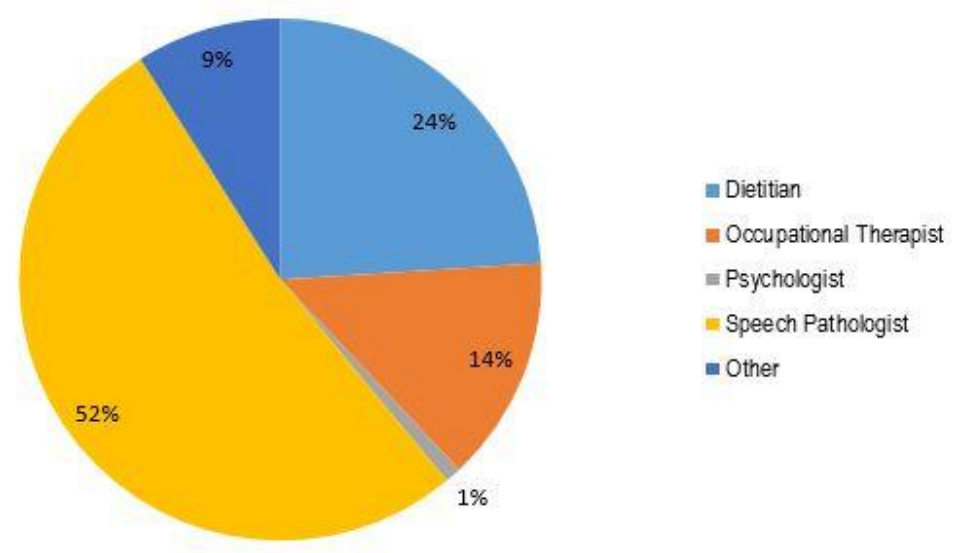

Figure 1: Respondents by profession

The majority $(52 \% ; n=52)$ of respondents had been working in their chosen profession for more than 10 years with $25 \%$ ( $n=25)$ working between $6-10$ years. These results aligned with the length of time that respondents had been working with children (defined as being less than 16 years of age). For the majority of respondents $(71 \% ; n=71)$ their caseload comprised $75-100 \%$ work with children.

\section{Feeding Caseload and Service Provision}

Of the total number of respondents, only $91 \%(n=91)$ completed the survey answering the questions relating to their work in the area of feeding and its relationship with the Guide. The majority of respondents providing information reported seeing children with feeding difficulties in early childhood at $0-2$ years $(n=78 ; 85 \%)$ and $3-5$ years $(n=68 ; 75 \%)$. Just over half of the respondents $(n=49 ; 54 \%)$ worked in a multidisciplinary feeding service.

Respondents rated their confidence in assessing a child with feeding difficulties in relation to clinical issues that align with the five assessment questions specified in the Guide. Responses $(n=85)$ are detailed in Figure 2 . Across professions, the majority were highly or somewhat confident in the determination of feeding safety $(n=52 ; 61 \%)$, assessment of feeding adequacy $(n=62 ; 73 \%)$, feeding efficiency $(n=59 ; 69 \%)$ and feeding skill development $(n=58 ; 68 \%)$. The remainder of clinicians rated themselves as not very confident or having no confidence.

\section{Implementation of the Guide}

The sections of the Guide with the highest reported use are displayed in Figure 3. There was an expected trend that the frequency of use of individual sections of the Guide was profession specific. For example, speech pathologists accessed the Guide more frequently for information related to safe swallowing. Occupational therapists accessed the Guide more frequently for information related to seating, positioning, and sensory processing. 


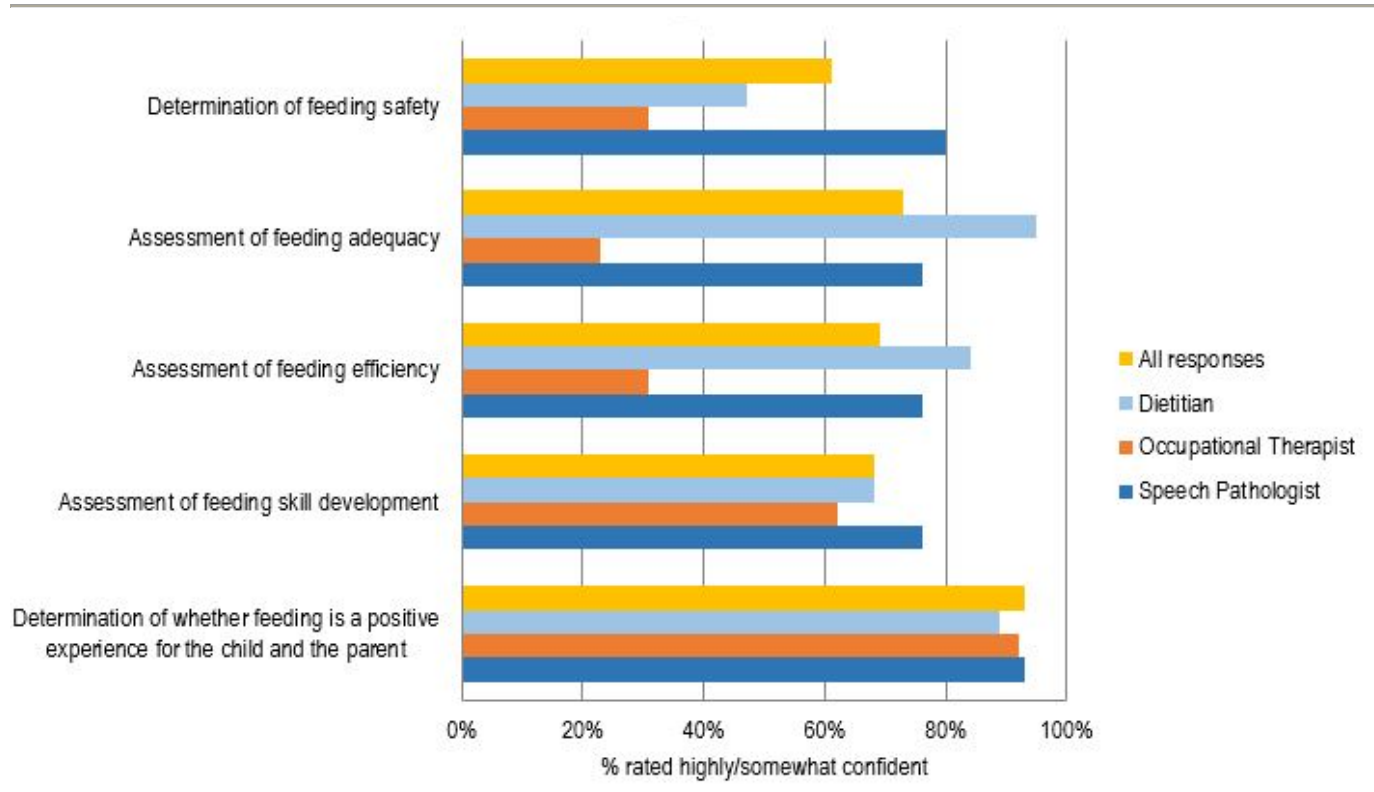

Figure 2. Self-rated confidence in assessment

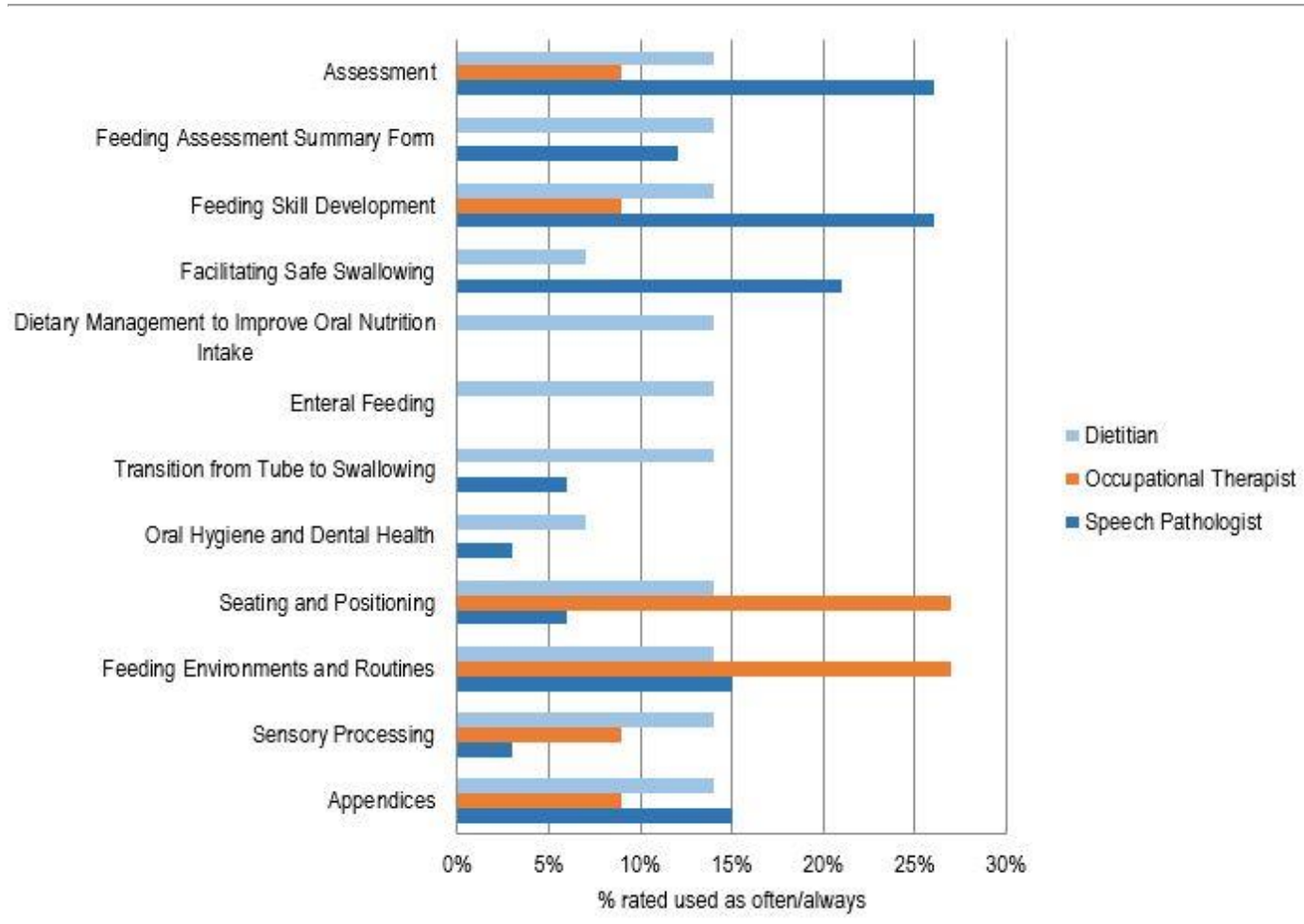

Figure 3: Most used sections of the Guide

Use of the Guide was one of the key themes identified in the focus group discussion. Overwhelmingly, focus group participants reported that they found the Guide to be a useful resource which could also support the education of students and less experienced clinicians. Participants indicated that the layout and format of the document made it easy to read. Consolidation of information across the broad spectrum of issues related to feeding difficulties into a single resource was also acknowledged as a strength of the document. These sentiments are highlighted by one respondent:

It's certainly very broad which is what you want for people to have more of that understanding, that holistic understanding of the realm of feeding across the broad spectrum of ages and disciplines involved across that whole child and family perspective. 
Participants most commonly reported use of the Guide to confirm the roles and scope of practice of the health professionals involved in the assessment and management of feeding difficulties. They referred to this content for their own information to guide and educate students, as well as less experienced clinicians and professional colleagues. Participants reported referring to content relating to the role of the professionals within a team to both validate and advocate for a multidisciplinary team approach for the management of feeding difficulties. One participant summarised this:

...it's more evidence behind us or more instruction to work in that multidisciplinary setting more than we have. Not saying that we haven't done it, but maybe we could make it more official now.

The assessment framework was highlighted as a component of the Guide that was of particular use. Participants reported using the five assessment questions, to structure or validate their own assessment processes. The framework was also used to support the clinical reasoning and problem solving of less experienced clinicians and students as they undertook assessment as well as to provide structure to their documentation and reports.

\section{Content of the Guide}

Participants' understanding regarding the scope of the Guide to support assessment and management was evaluated using a case study. Only $44 \%$ ( $n=59)$ of respondents reporting use of the Guide completed questions relating to the case study. All of these respondents correctly indicated that the Guide would be relevant and provided comments to support their answer related to the themes illustrated in Table 1.

Table 1: Reasons for using the Guide

\begin{tabular}{|l|l|}
\hline Reasons for using the Guide & Number (\%) \\
\hline Framework to support clinical reasoning & $16(27 \%)$ \\
\hline Information regarding a range of factors & $16(27 \%)$ \\
\hline Useful for both complex and non-complex cases & $8(14 \%)$ \\
\hline Information and strategies to support management & $7(12 \%)$ \\
\hline
\end{tabular}

Multiple choice questions used to evaluate participants' understanding of the content from various sections of the Guide were completed by $67(57 \%)$ respondents. The results are detailed in Figure 4 . A relationship between respondents' demographics and their uncertainty regarding the correct answer was not significant for any of the questions posed.

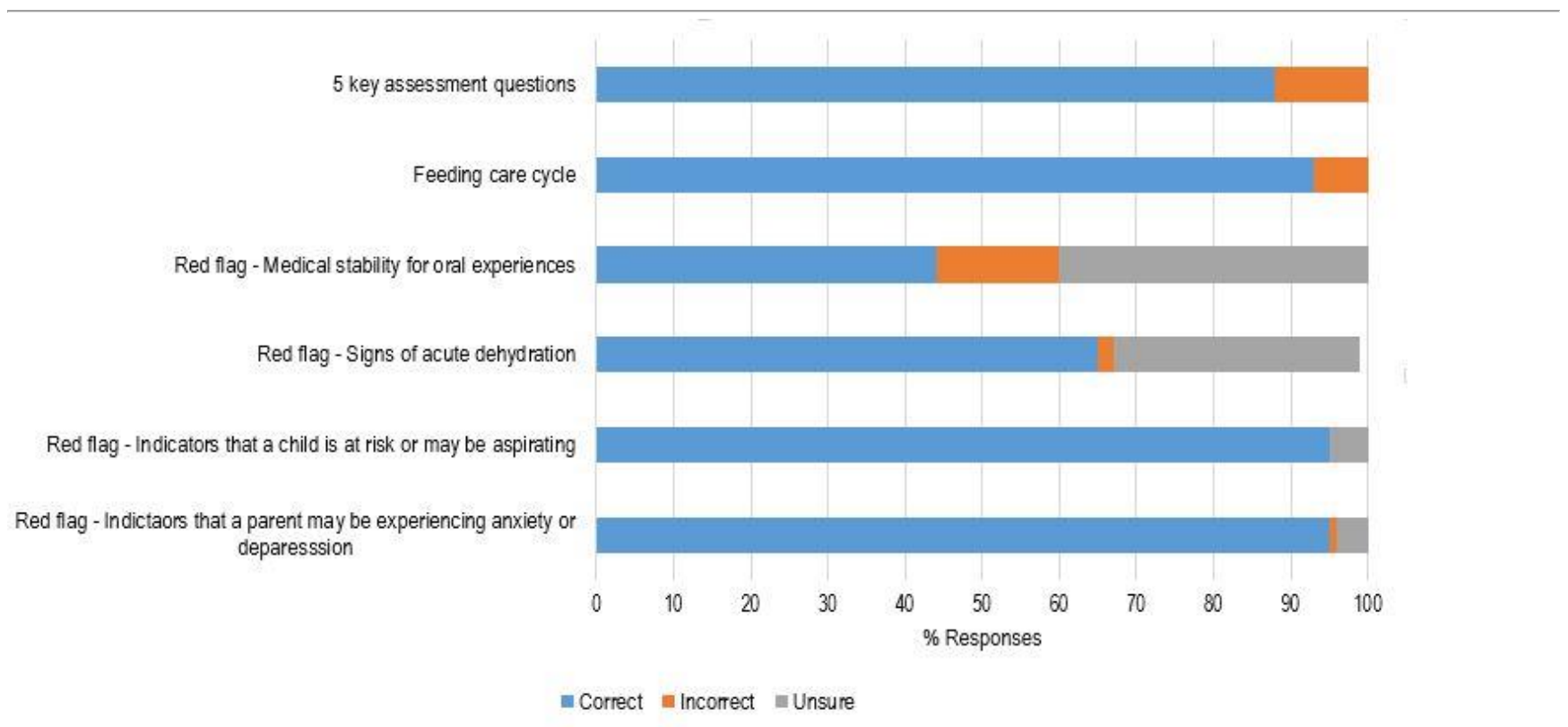

Figure 4: Understanding of the Guide content

Focus group participants suggested a number of areas for improvement in the Guide with one being the inclusion of parent handouts or additional links to information for parents. Suggested topics included pictures of different fluid consistencies and food textures as well as tips for how to introduce new foods. It was indicated that the images of food textures would also be of use to health professionals using the Guide. The limitations of regularly updating the content of the Guide, such as feeding team details and references, were discussed. One 
recommendation was the establishment of an online portal to accompany the Guide, to house recently published materials, updated references and feeding team details.

\section{Impact of the Guide}

Respondents $(n=76 ; 89 \%)$ rated how satisfied they were that the Guide provided an effective framework for the management of children with feeding difficulties. Figure 5 illustrates the satisfaction with the Guide by professional group.

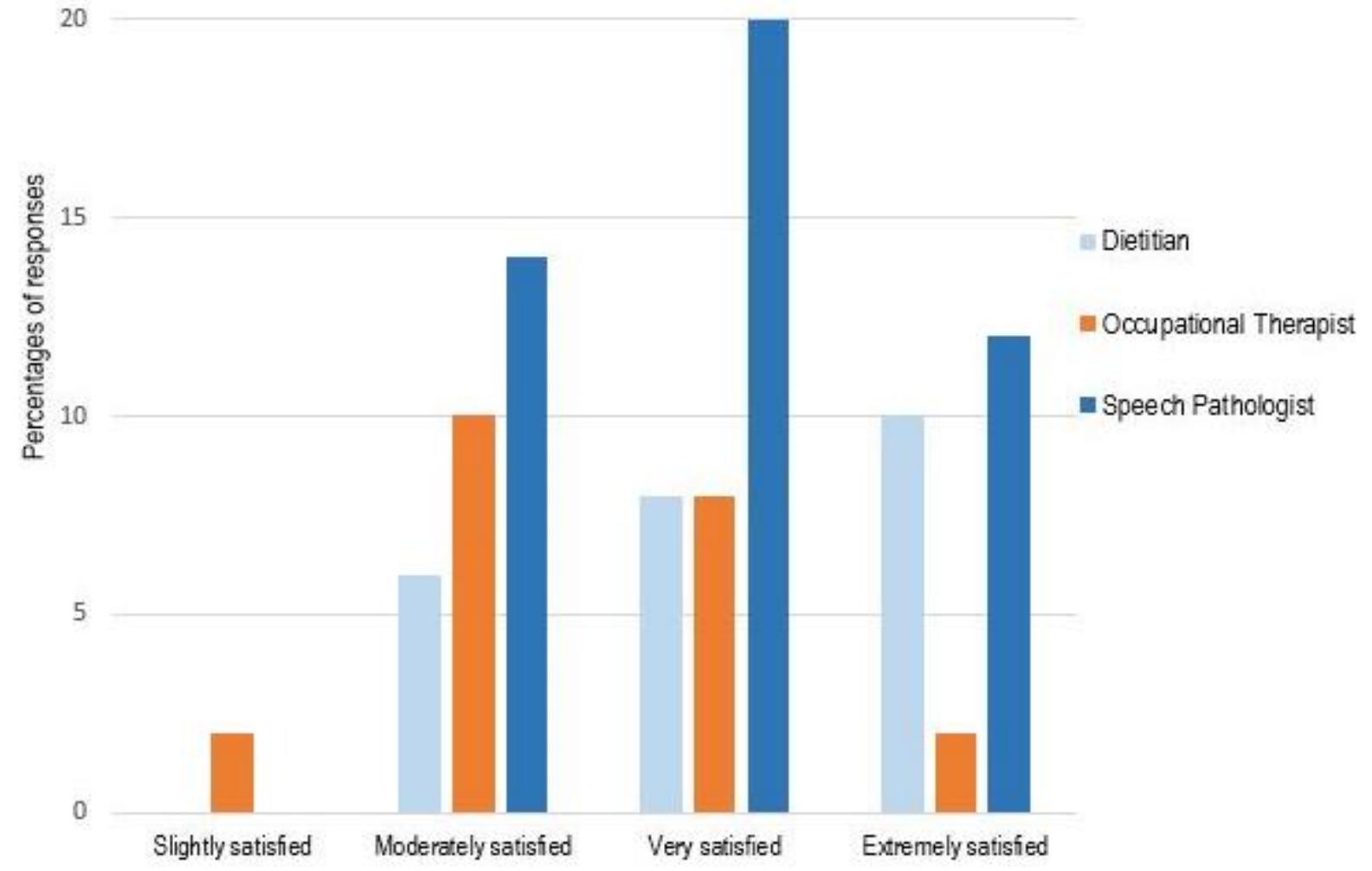

Figure 5: Satisfaction with the Guide by profession

Participants ( $n=31$ ) identified changes to their practice since the Guide was released. Responses referred to a range of common themes detailed in Table 2. A number of professionals indicated nil or minimal change to their practice $(n=7 ; 23 \%)$. These themes identified are listed in Table 2.

Table 2: Changes to practice since the Guide was released

\begin{tabular}{|l|l|}
\hline Changes to Practice & Number (\%) \\
\hline Support to the structure of assessment and management & $9(29 \%)$ \\
\hline Knowledge of what resources were available and where to find them & $4(13 \%)$ \\
\hline Provision of a sense of completion or being thorough & $4(13 \%)$ \\
\hline Support clinical reasoning & $3(10 \%)$ \\
\hline Increase of confidence & $3(10 \%)$ \\
\hline Provision of greater assistance to the multidisciplinary team & $2(6 \%)$ \\
\hline Teaching / training & $2(6 \%)$ \\
\hline
\end{tabular}

Focus group participants identified the most significant changes in practice related to the application of the assessment framework. In addition to providing a more standardised or formalised assessment process, participants reported use of the assessment questions to document and report assessment results. This included the opportunity to use a consistent structure and language in this communication as highlighted by one respondent: 
Those 5 questions as part of the assessment process has helped me in my communication with other team members, to sort of frame it really well. To think about if I'm seeing a patient in isolation and have concerns, how I actually present those problems to my colleagues.

A number of participants also indicated that the assessment framework was used during intake to obtain information and assist with prioritisation of referrals.

Conversely, participants were asked to identify barriers to using the Guide. The two main themes identified were access to a hard copy of the Guide $(19 \%)$ and time to review the document, develop related resources and provide recommended services (17\%). A number of respondents $(19 \%)$ of respondents identified there were no barriers to using the Guide.

Finally, respondents were asked to identify additional education and support to enable use of the Guide. Participants were able to select as many answers as they felt were relevant. Responses are listed in Table 3. A number of participants $11 \%(n=8)$ identified that no further education or clinical support was necessary.

Table 3: Additional education support to facilitate use of the Guide

\begin{tabular}{|l|l|}
\hline Additional Education Support & Number (\%) \\
\hline Paediatric feeding support network & $44(58 \%)$ \\
\hline Online learning module & $43(57 \%)$ \\
\hline Allied Health Telehealth Virtual Education session & $39(51 \%)$ \\
\hline Face to face workshop & $32(42 \%)$ \\
\hline Educational secondment & $24(32 \%)$ \\
\hline
\end{tabular}

Promotion of the Guide was recommended as the best way to support its use. Strategies for promotion included linking with regional advisors to develop local support, or establishing local champions. Incorporating references to the Guide into local orientation, ongoing clinical supervision and accreditation procedures as well as the role of senior clinicians in supporting the use of the Guide was emphasised.

Support from service managers in local facilities was identified as an important factor in encouraging allied health professionals to use the Guide and adopt the recommended changes in practice. This included supporting clinician time, allocation of resources, and incorporating the Guide into relevant policies and procedures. The role of larger facilities in promoting the uptake of the Guide was also acknowledged by a number of participants. This is highlighted in the following:

If management in bigger hospitals approve it, we get a stronger support in terms of setting up bigger/more enhanced feeding clinics, rather than it being driven by a clinician, bottom-up push.

In addition to supporting use of the Guide, focus group participants discussed the best way to upskill allied health professionals in preparation for working with children with feeding difficulties.

The establishment of clinical support networks, both formal and informal, was the most common recommendation. While participants made reference to support from the tertiary children's hospitals, there was a particular emphasis on the value of local support networks. This could comprise one or a combination of phone consultations, outreach support by a clinical senior, site visit/work shadowing in a feeding clinic (multidisciplinary), joint appointments in the local facility, and networking with local non-government organisations.

Participation in work shadowing opportunities was recommended as an effective education strategy by numerous focus group members. This included formal educational secondment visits to the tertiary children's hospitals or specialist feeding clinics. Likewise, opportunities to shadow more experienced clinicians in their own facility or visit local established feeding clinics were considered valuable. In addition to consolidating the clinician's understanding of their own profession, these experiences may also be used to promote the role of other professions.

Shared care between facilities in a LHD was a strategy proposed in one of the focus groups. Participants in this group identified the challenge of upskilling clinicians who may be required to manage children with feeding difficulties on an irregular basis. In this way, shared care was considered a way of supporting clinicians to manage individual clients, rather than more broadly increasing their competence as the participant below highlighted:

Just that option of coming once with the child that you're seeing. Even to get set-up with the right tools and equipment and you become more aware of the child. It might be another 10 years before you see another child like that. So, it's not even necessarily about upskilling their local therapists, it's about knowing where to come at the time. 


\section{DISCUSSION}

Data collected through the questionnaire and focus group discussion provides a robust overview of how the Guide has been utilised across NSW. The main findings of the evaluation are discussed under five themes; i) characteristics of respondents and their teams, ii) use of the Guide, iii) knowledge of content, iv) satisfaction with the Guide, v) future directions.

\section{Characteristics of Respondents and Their Teams}

Respondents comprised the six allied health professions that are predominantly involved in the assessment and management of paediatric feeding difficulties i.e. dietetics, occupational therapy, physiotherapy, psychology, social work and speech pathology. The majority of responses were received from speech pathologists and dietitians which aligned with the high prevalence of these professions on feeding teams. The composition of the feeding team varied across the different settings, and included allied health professionals working in isolation as well as those working in interprofessional teams. Service delivery models varied across the different contexts. The majority of respondents reported that they were highly or somewhat confident in relation to the five assessment questions specified in the Guide. This confidence varied across the professional groups and tended to be closely related to their respective scope of practice.

\section{Use of the Guide}

One of the primary uses of the Guide reported was as a tool to consolidate existing knowledge of paediatric feeding. The five key assessment questions and the paediatric feeding care cycle contained within the Guide added clarity and structure to assessment processes. Whilst the Guide did not offer new content to many participants, it provided a standardised language and framework that allowed more fluid communication and, a framework for education, prioritisation of referrals, decision making, and a tool for emphasising the importance of paediatric feeding within their service. For other participants, it reinforced that paediatric feeding is a clinically complex area and provided an opportunity to reflect on practice and consider the assessment process in a different way i.e., as a cycle that emphasised re-assessment.

\section{Knowledge of Content}

It must be noted that the number of respondents answering the questions assessing knowledge of the content of the Guide was lower than other responses on the questionnaire. There are no identifiable reasons for the lower numbers, however, conjecture may include uncertainty with answers or reluctance to complete exam type questions.

From the responses provided, questions relating to red flags concerning medical stability and dehydration indicated the lowest rates of correct answers. This indicated a potential gap in knowledge. Respondents were more confident in identifying how they would respond to parental anxiety and depression.

Notwithstanding the comprehensive information and complementary resources in the Guide, participants acknowledged the necessity for actual work experience to increase competency in the management of feeding difficulties in children.

\section{Satisfaction with the Guide}

Overall the Guide was valued with respondents indicating they found it easy to use. The profession specific information contained was said to be beneficial not only for the Guide but also for the variety of uses it provided. There was variation in how respondents rated their frequency of use for each section of the Guide as different elements are more relevant for particular health professionals. The broad scope of the Guide, however, was seen as a positive aspect and aligned with the primary aims, enabling clinicians to obtain a holistic understanding of paediatric feeding across ages and professions.

\section{Future Directions}

Moving forward the Guide will be embedded into a broader education strategy. This will be translated to various modalities including recorded, eLearning, factsheets or self-paced modules that offer flexibility and include case studies. In light of the described benefits of shared care of children with feeding difficulties, for example, between regional and rural centres, or tertiary and local centres, it is essential to facilitate this moving forward. The potential to utilise educational secondments, not just to the tertiary children's hospitals but also to regional centres where established teams could assist in the up skilling of allied health professionals were identified. The concept of utilising local support networks, shared cared and educational secondments in conjunction with the use of the Guide, as a supporting document, would facilitate development of skills and confidence.

\section{Limitations}

A major limitation of the evaluation was the low response rate to the questions assessing knowledge of the content of the Guide. There would appear to be no obvious reasons for the lower numbers; however, conjecture may include uncertainty with answers or reluctance to complete exam type questions. The results of the evaluation therefore only reflect self-reported change in practice and improvement in knowledge. 


\section{CONCLUSION}

The evaluation of the Guide indicated that it is viewed as a useful tool by allied health professionals working with children with feeding difficulties. Experienced respondents reported that the Guide provided validation for current practice, whereas those with less experience indicated it supported them in their work within this clinically complex realm. Further promotion of the Guide and education regarding its use will assist in informing the assessment and management of feeding difficulties in children. This education should take the form of a range of modalities and be supported by the development of clinically relevant resources.

\section{REFERENCES:}

1. Office of Kids and Families. Feeding difficulties in children: A guide for allied health professionals. 2016. Available from: https://www1.health.nsw.gov.au/pds/ActivePDSDocuments/GL2016_007.pdf.

2. Bryant-Waugh R, et al. Feeding and eating disorders in childhood. International Journal of Eating Disorders. 2010;43(2):98-111.

3. Arvedson J. Assessment of pediatric dysphagia and feeding disorders: Clinical and instrumental approaches. Developmental disabilities research reviews. 2008;14:118-27. doi: 10.1002/ddrr.17.

4. Kerzner B, Milano K, MacLean W, Berall G, Stuart S, Chatoor I. A Practical Approach to Classifying and Managing Feeding Difficulties. Pediatrics. 2015;135(2):344-353. doi: 10.1542/peds.2014-1630.

5. Williams S, et al. Pediatric feeding and swallowing problems: an interdisciplinary team approach. Can J Diet Pract Res, 2006;67(4):185-90.

6. Woolf S, Grol R, Hutchinson A, Eccles M, Grimshaw J. Potential benefits, limitations, and harms of clinical guidelines. BMJ.1999;318(7182):527-530.

7. O'Leary S. The Essential Guide to Doing Your Research Project. 2nd.ed. London: Sage; 2014.

8. DePoy E, Gitline L. Introduction to Research St Louis: Mosby; 1984.

9. Rattray J, Jones MC. Essential elements of questionnaire design and development. J Clin Nurs. 2007;16(2):234-243. doi:10.1111/j.1365-2702.2006.01573.x

10. DeJonckheere M, Vaughn LM. Semistructured interviewing in primary care research: a balance of relationship and rigour Family Medicine and Community Health 2019;7:e000057. doi: 10.1136/fmch-2018-000057

11. Braun V, Clarke V. Using thematic analysis in psychology. Qualitative Research in Psychology. 2006;3:77-101. 